\title{
Flexible x-ray source with tunable polarization and orbital angular momentum from Hermite-Gaussian laser modes driven plasma channel wakefield
}

\author{
B. Lei, ${ }^{1,2, *}$ T. Teter, ${ }^{1,2}$ J. W. Wang, ${ }^{3}$ V. Yu. Kharin, ${ }^{1}$ C. B. Schroeder, ${ }^{4}$ \\ M. Zepf, ${ }^{1,2}$ and S. G. Rykovanov ${ }^{1,5, \dagger}$ \\ ${ }^{1}$ Helmholtz Institute Jena, Fröbelstieg 3, 07743 Jena, Germany \\ ${ }^{2}$ Faculty of Physics and Astronomy, Friedrich-Schiller-Universität Jena, 07743 Jena, Germany \\ ${ }^{3}$ Shanghai Institute of Optics and Fine Mechanics, Chinese Academy of Sciences, 201800 Shanghai, China \\ ${ }^{4}$ Lawrence Berkeley National Laboratory, Berkeley, California 94720, USA \\ ${ }^{5}$ Center for Computational and Data-Intensive Science and Engineering, \\ Skolkovo Institute of Science and Technology, Moscow 121205, Russia
}

(Received 14 January 2019; published 29 July 2019)

\begin{abstract}
A plasma channel undulator/wiggler may be created through the plasma wakefield excited by the beating of several Hermite-Gaussian laser modes propagating in a parabolic plasma channel. Control over both the betatron and undulator forces is conveniently achieved by tuning the amplitude ratios, colors, and order numbers of the modes. A special structure of the undulator/wiggler field without the focusing force near the propagation axis is generated inside the plasma wakefield by matching the strengths of the fundamental and first-order Hermite-Gaussian modes. The electron beam only experiences forced undulator oscillations in such a field, which significantly improves the quality of the emitted radiation. Since the value of the undulator strength parameter could be in a wide range, less or larger than unity, it is capable of generating narrow bandwidth $\mathrm{x}$-ray, as well as the synchrotronlike high-energy $\mathrm{x} / \gamma$-ray, radiation by harmonics. Additionally, controlling the relative phases between the laser modes allows for polarization control of the plasma undulator. High-order harmonics produced from a circularly polarized plasma undulator clearly show the vortex nature and carry well-defined orbital angular momentum.
\end{abstract}

DOI: 10.1103/PhysRevAccelBeams.22.071302

\section{INTRODUCTION}

Extreme ultraviolet (XUV) and x-ray pulses produced by electrons traveling through magnetic undulators have become an indispensable tool for experimental studies in chemistry, biology, material science, warm dense matter, etc. The wavelength of the on-axis radiation generated by an electron with relativistic Lorentz factor $\gamma$ traveling through a linearly polarized undulator is equal to $\lambda=$ $\lambda_{u}\left(1+a_{u}^{2} / 2\right) /\left(2 \gamma^{2}\right)$, where $\lambda_{u}$ is the undulator period, $a_{u}=$ $e B_{u} \lambda_{u} /\left(2 \pi m_{e} c\right)$ is the undulator strength, $B_{u}$ is the magnetic field amplitude, $e$ and $m_{e}$ are the absolute values of electron charge and mass respectively, and $c$ is the speed of light in vacuum. For optimal operation of undulator radiation or free-electron laser (FEL) facilities, it is important to have both high undulator strength (on the order of

\footnotetext{
*B.Lei@gsi.de

S.Rykovanov@gsi.de
}

Published by the American Physical Society under the terms of the Creative Commons Attribution 4.0 International license. Further distribution of this work must maintain attribution to the author(s) and the published article's title, journal citation, and DOI. unity) and small undulator period. Currently, the technological limit for the magnetic undulators, capable of achieving $a_{u} \sim 1$, is on the order of several centimeters. Decreasing the undulator period will lead to smaller and cheaper synchrotron facilities and is therefore highly desired. This is especially true in view of the recent tremendous progress in laser plasma accelerators (LPAs), where quasimonoenergetic electron beams with $\sim 1 \%$ energy spread in the $\mathrm{GeV}$ range were experimentally demonstrated [1-5]. Electron acceleration happens on the scale of centimeters and hence the footprint of the LPA is the driving laser system itself, which (for many applications) can be as compact as $6 \mathrm{~m}^{2}$. The total size of the facility using an LPA and magnetic undulator will be, in a general case, dictated not by the size of the accelerator, but by the size of the electron optics (conventionally several meters for magnetic quadrupole lenses) and the undulator (conventionally several tens of meters or more). It has recently been experimentally demonstrated that electron optics can, in principle, also be replaced by plasma technology [6-8], leaving the undulator as the only major factor defining the size of the facility. Several ideas for undulators with periods on the order of millimeter or below 
(often referred to as microundulators) have been proposed, including electrostatic $[9,10]$, crystalline [11-13], microwave [14], plasma [15], nano-wire [16] and laserbased [17-25].

A twisted photon beam carrying orbital angular momentum (OAM) can be obtained from a single free electron in circular or spiral motion [26-29]. Such a beam is capable of interacting with particular atomic orbitals which are defined by the angular momentum associated with the particles orbit in atoms [30], molecules [31], solid state systems [32] and plasma [33]. Radiation sources with known OAM enable the probing of specific atomic structures. Applications of $\mathrm{x}$-ray beams with internal OAM include probing quantum optics [34], optical spanners [35], and crystalline structures [36].

Recently, a novel plasma undulator concept for narrowbandwidth photon emission was proposed. The plasma undulator or wiggler (PIGGLER) is based on wakefields created by the laser pulse undergoing centroid oscillations in a plasma channel [37-40]. Detailed linear theory for the case of a Gaussian laser pulse injected with some initial offset and/or with some initial angle relative to the plasma channel symmetry axis has been developed [37,41]. It has been shown, that an electron beam correctly injected in the wakefield structure oscillates with the characteristic undulator wavelength equal to $\lambda_{u}=2 \pi Z_{R}$, where $Z_{R}$ is the Rayleigh length of the laser pulse. The main contribution to the broadening of the radiated photon spectrum was identified to be the electron beam divergence, which is rather large due to strong focusing inside the wakefields. It was also proposed to extend the spectrum to the synchrotronlike $\gamma$-ray radiation by using the resonance between the centroid oscillation of laser pulse and the betatron oscillation of electrons [40]. The oscillation of the electron beam is increased in the early resonant stage and then becomes semi-stable after sufficient energy gain from the wakefield. The resonant scheme is capable of enhancing the brightness of the emitted photon beam due to the large field volume. It was also mentioned that using the superposition of two (odd and even) higher-order Hermite-Gaussian modes may (1) lead to a better control over the strength of the focusing forces, relaxing the conditions for the electron beam emittance for generation of a narrow-bandwidth photon spectrum; and (2) provide more control for the parameters of the plasma undulator (its strength and wavelength). It is the purpose of this paper to extend previous work $[37,39,41]$ and provide detailed analytical derivations for the case where the plasma undulator is created by the superposition of higher-order laser modes propagating inside the parabolic plasma channel. It will also show that our scheme is capable of generating harmonics carrying OAM in the circularly polarized undulator.

The paper is organized as follows: In Sec. II, propagation of a Hermite-Gaussian laser pulse in a plasma channel is discussed. It is shown that the oscillation of the pulse is due to the interference between different modes. In Sec. III, the wakefield structure of a plasma undulator/wiggler is derived by using linear laser-plasma theory. Polarization is discussed and the initial condition given by the strength of laser pulses is found to generate a special structure of wakefield without the focusing force near the propagation axis. In Sec. IV, the dynamics of a single relativistic electron and an electron beam are studied. Radiation calculation is presented in Sec. V.

\section{LASER PULSE PROPAGATION INSIDE A PARABOLIC PLASMA CHANNEL}

Laser pulse guiding inside the parabolic plasma channels $[42,43]$, for example as created by capillary discharges, is routinely performed in LPA experiments $[3,44,45]$ in order to avoid laser pulse diffraction and, thus, maintain a high acceleration gradient $(\sim 1 \mathrm{GeV} / \mathrm{cm})$ for several centimeters.

The equation governing the dynamics of the slowly varying envelope $\tilde{a}_{\perp}(\tau, x, y)$ of the laser pulse inside the parabolic plasma channel within the paraxial approximation is given by the Schrödinger-like equation for a harmonic oscillator

$$
i \frac{\partial \tilde{a}_{\perp}}{\partial \tau}=\left[\frac{\hat{p}^{2}}{2 M_{p}}+V(\rho)\right] \tilde{a}_{\perp}=\hat{H} \tilde{a}_{\perp}
$$

where $\hat{p}=-i \nabla_{\perp}$ is the momentum operator and $V(\rho)=$ $\left(1 / M_{p}^{2}+\Omega_{H}^{2} \rho^{2}\right) M_{p} / 2$ is the harmonic oscillator potential with the eigenfrequency $\Omega_{H}=\left(M_{p} R\right)^{-1}$ and $\rho^{2}=x^{2}+y^{2}$. Equation (1) is written using normalized space-time coordinates: $\tau=\omega_{p} t^{\prime}, x=k_{p} x^{\prime}, y=k_{p} y^{\prime}, z=k_{p} z^{\prime}$, with the plasma wave number $k_{p}=\omega_{p} / c=\left(4 \pi r_{c l} n_{0}\right)^{1 / 2}$, where $n_{0}$ is on-channel-axis plasma density and $r_{c l}=e^{2} / m_{e} c^{2}$ is the classical electron radius. The "effective mass" of the oscillator is defined as $M_{p}=k_{L} / k_{p}$, and is equal to the ratio of laser pulse and plasma wave numbers. The normalized channel radius is defined as $R=r_{m}^{2} / 2$, where $r_{m}=k_{p} / \sqrt{\pi r_{c l} \Delta n}$ is the dimensionless, matched laser spot size and $\Delta n$ is the channel depth, so that the channel density profile can be written as $n(\rho)=$ $n_{0}+\rho^{2} \Delta n / R^{2}$. The dimensionless laser pulse vector potential is then given by $a_{\perp}(\tau, \rho, \zeta)=e A_{\perp} / m_{e} c^{2}=$ $1 / 2 \tilde{a}_{\perp}(\tau, \rho) g(\zeta) \exp \left(i M_{p} \zeta\right)+$ c.c., where $A_{\perp}$ is the transverse laser pulse vector potential in Gaussian units, $g(\zeta)$ is the laser pulse temporal envelope (normalized to 1 ) and $\zeta=k_{p}\left(z^{\prime}-c t^{\prime}\right)$. In writing Eq. (1), it was also assumed that laser pulse vector potential amplitude $a_{0}<1$, and that self-focusing is not important, which is valid if the power of the laser pulse satisfies $P<P_{c}$, where $P_{c}[G W] \simeq 17 M_{p}^{2}$.

The general solutions of the Schrödinger equation (1) for the 2-dimensional harmonic oscillator in Cartesian geometry are well known [46]. A propagating, linearly polarized 
laser pulse can be written as a Hermite-Gaussian mode of order $m$ and $n$ as follows

$$
\tilde{a}_{\perp}(\tau, x, y)=\frac{a_{0}}{2} \eta \chi(x, y) e^{-i \theta \tau+i \varphi},
$$

where $a_{0}$ is the normalized amplitude of the mode and $\varphi$ is its phase. $\eta=1 / \sqrt{2^{m+n} m ! n !}$ is normalization coefficient, where $m$ and $n$ are the numbers of modes in $\hat{x}$ and $\hat{y}$ directions respectively. $\chi(x, y)=H_{m}(x / \sqrt{R}) H_{n}(y / \sqrt{R})$ $\exp \left\{-\left(x^{2}+y^{2}\right) / 2 R\right\}, \quad \theta=\left(1 / 2 M_{p,(m, n)}+(m+n+1) /\right.$ $\left.M_{p,(m, n)} R\right)$, where $H_{n}(x)$ is a Hermite polynomial of $n$th order, and $M_{p,(m, n)}$ is introduced to take into account that modes can have different wavelengths.

Modes given by Eq. (2) represent the stationary states and propagate along the channel with constant centroid position and with constant spot size. Dynamics of any laser pulse, propagating along the channel, can be represented as the superposition of different Hermite-Gaussian modes given by Eq. (2), i.e., $a_{\perp}(\tau, x, y, \zeta)=\sum_{\nu} a_{\perp, \nu}(\tau, x, y, \zeta)$, where $a_{\perp, \nu}(\tau, x, y, \zeta)$ represents the $\nu$ th pulse. As discussed in [47], it is beneficial to use a superposition of different Hermite-Gaussian modes for controlling the focusing force of the plasma wakefields, which might help to reduce electron beam divergence. In the case of the superposition of various Hermite-Gaussian modes, due to the difference in the phase velocity, the intensity profile $I=a_{\perp}(\tau, x, y, \zeta) a_{\perp}(\tau, x, y, \zeta)^{*}$ will contain interference terms and can lead to the oscillatory structure which is dependent on the mode numbers. The oscillation frequency induced by two pulses of different modes, denoted by $\nu$ and $\lambda$, is $\Omega_{\nu, \lambda}=\Delta \theta_{\nu, \lambda}=\theta_{\nu}-\theta_{\lambda}$. With $n$ laser pulses, the oscillation amplitude is increased by $n ! / 2$ in the resonant case if $\Omega_{\nu, \lambda}=\Omega$ is the same for every set of two different modes.

Such oscillation can be seen on Fig. 1, where the integrated transverse intensity profile of the superposition of the mixture of modes with identical $M_{p}$ propagating along the plasma channel is demonstrated for three cases: (1) superposition of two laser modes with $\{m=0, n=0$, $a=0.4, \varphi=0\} \quad$ and $\quad\{m=1, n=0, a=0.05, \varphi=0\}$ [subfigure (a)]; (2) superposition of two laser modes with $\{m=0, n=0, a=0.4, \varphi=0\}$ and $\{m=3, n=0, a=0.05$, $\varphi=0\}$ [subfigure (b)]; and (3) superposition of three laser modes with $\{m=0, n=0, a=0.4, \varphi=0\},\{m=1, n=0$, $a=0.05, \varphi=0\}$ and $\{m=0, n=1, a=0.05, \varphi=\pi / 2\}$ [subfigure (c)]. The third case can be also viewed as the superposition of a Gaussian mode and a first order Laguerre-Gaussian mode in the case of only two pulses being required. In cases (1) and (2), the oscillation is linear in the $\hat{x} \hat{z}$ plane. In case (3), the oscillation is helical. One can see a behavior that is very similar to the case of a Gaussian laser pulse injected into the parabolic channel with some initial centroid displacement or with some angle with respect to the channel axis, which is discussed in detail
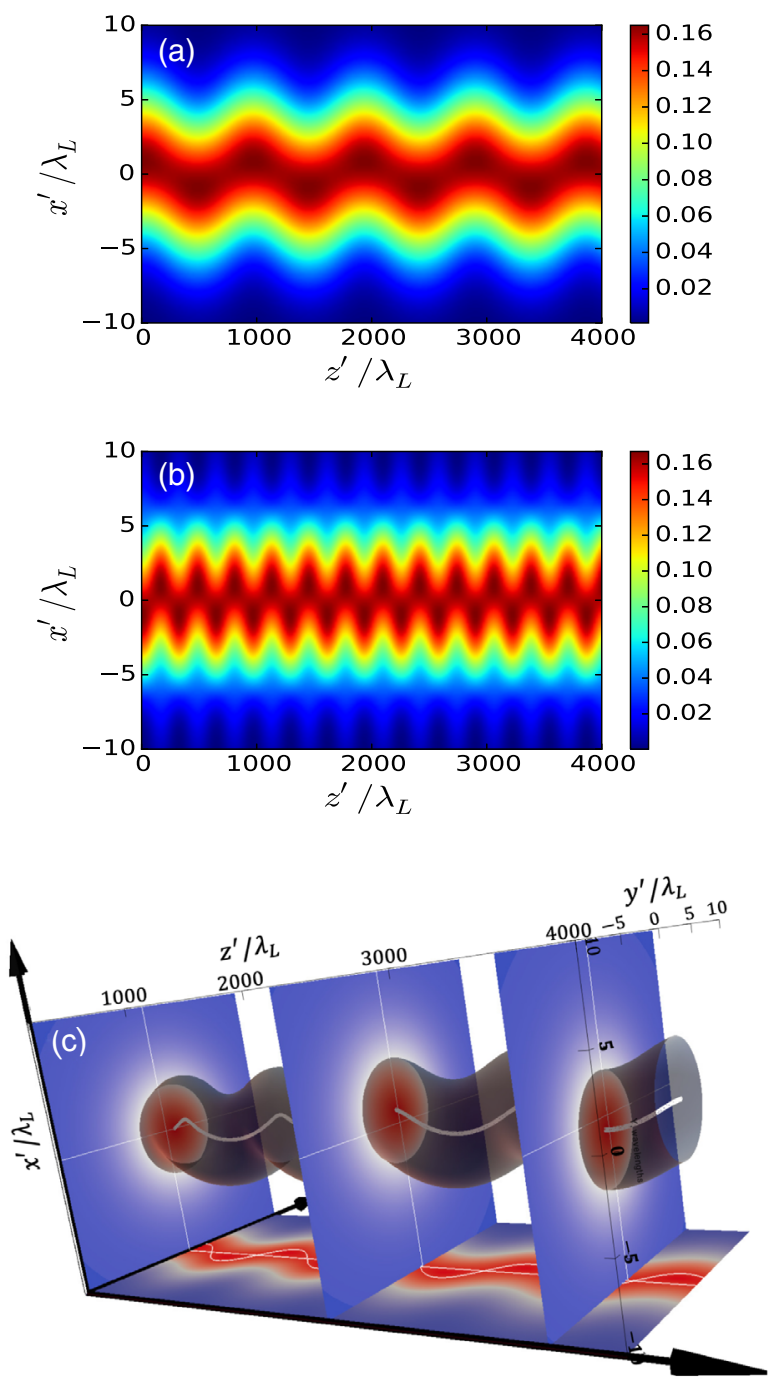

FIG. 1. Integrated transverse intensity profile of laser pulse as a function of propagation distance $Z^{\prime} / \lambda_{L}$ for (a) a fundamental mode plus a first-order Hermite-Gaussian mode; (b) a fundamental mode plus a third-order Hermite-Gaussian mode; (c) a fundamental mode plus two first-order Hermite-Gaussian modes.

in previous works [37,40,41,47]. From Eq. (2), one can also find that by choosing different mode numbers, amplitudes, phases, and wavelengths, one can create oscillatory behavior in the propagating laser pulse with different periods and amplitudes. Ellipticity of the trajectory of the laser pulse centroid can be controlled by the phase difference of the laser modes, as can be seen on Fig. 1(c). This provides an easy control of the polarization of the plasma channel undulator based on higher-order laser modes. The plasma wakefield structure excited by the beating of two propagating laser modes will be discussed in Sec. III.

\section{UNDULATOR FIELDS STRUCTURE}

Undulator field structure, i.e., the structure of the excited plasma wakefield, can be calculated from the propagation 
of the laser pulse (here and further, by the laser pulse, a mixture of several higher-order Hermite-Gaussian modes is to be understood). The equation for the dimensionless scalar potential $\phi=e \Phi / m_{e} c^{2}$ is [43]:

$$
\frac{\partial^{2} \phi}{\partial \zeta^{2}}+\phi=\frac{a_{\perp} a_{\perp}^{*}}{2}
$$

where $\Phi$ is the scalar potential in Gaussian units and the following assumptions have been employed: (1) the laser pulse intensity is nonrelativistic, i.e., $a_{0}<1$ so that linear plasma theory can be applied $[43,48]$; (2) the laser pulse propagates near the speed of light in a vacuum; (3) the plasma channel is broad, and the effect of channel curvature on the wakefield is negligible. Equation (3) has the following solution [43]:

$$
\phi(\tau, x, y, \zeta)=\int_{-\infty}^{\zeta} d \zeta^{\prime} \sin \left(\zeta-\zeta^{\prime}\right) \frac{a_{\perp}\left(\zeta^{\prime}\right) a_{\perp}^{*}\left(\zeta^{\prime}\right)}{2}
$$

which depends on the intensity structure of the laser pulse and $a_{\perp}(\zeta)=\sum_{\nu} \tilde{a}_{\perp, \nu}(\tau, x, y) g_{\nu}(\zeta) \exp \left(i M_{p, \nu} \zeta\right)$, where $\tilde{a}_{\perp, \nu}$ represents the vector potential of the $\nu$ th mode with mode numbers $m_{\nu}$ and $n_{\nu}$ and $M_{p, \nu}$ represents its "mass".

Consider a laser pulse representing the beating of several different modes. In this case, the right-hand side (r.h.s.) of Eq. (3) can be written as

$$
\frac{a_{\perp} a_{\perp}^{*}}{2}=\frac{1}{4} \sum_{\nu}\left|\tilde{a}_{\perp, \nu}\right|^{2} g_{\nu}^{2}+\frac{1}{2} \sum_{\nu \neq \lambda} \operatorname{Re}\left\{\tilde{a}_{\perp, \nu} \tilde{a}_{\perp, \lambda}^{*}\right\} g_{\nu} g_{\lambda},
$$

The second term, interference between every set of two different modes, in r.h.s of Eq. (5) is nonzero if the modes have parallel polarizations [47].

Assuming beating of $N$ laser pulses with the different modes and the same longitudinal size, $w_{z, \nu}=w_{z}$, and then $g_{\nu}^{2}(\zeta)=e^{-2 \zeta^{2} / w_{z}^{2}}$, for the solution of $\phi$, one can write

$$
\begin{aligned}
\phi(\zeta, x, y)= & C_{\beta} \sin \zeta \sum_{\nu=1}^{N} a_{\nu}^{2} \eta_{\nu}^{2} \chi_{\nu}^{2} \\
& +\sin \zeta \sum_{\nu \neq \lambda}^{N} C_{u, \nu, \lambda} a_{\nu} a_{\lambda} \eta_{\nu} \eta_{\lambda} \chi_{\nu} \chi_{\lambda} \\
& \cdot \cos \left(\Omega_{\nu, \lambda} \tau-\Delta \varphi_{\nu, \lambda}\right),
\end{aligned}
$$

where $\quad C_{\beta}=\frac{1}{4} \sqrt{\frac{\pi}{2}} w_{z} e^{-w_{z}^{2} / 8}, \quad C_{u, \nu, \lambda}=C_{\beta} e^{-\Delta M_{p, \nu, \lambda}^{2} w_{z}^{2} / 8}$ $\cosh \frac{\Delta M_{p, \nu, \lambda} w_{z}^{2}}{4} . \Delta M_{p, \nu, \lambda}=M_{p, \nu}-M_{p, \lambda}$ describes the possible differences of modes' wavelengths. $\Omega_{\nu, \lambda}=\theta_{\nu}-\theta_{\lambda}$ is the term describing the oscillation frequency of a beating wave due to the interference of two different modes and $\Delta \varphi_{\nu, \lambda}=\varphi_{\nu}-\varphi_{\lambda}$ describes the phase difference. The second, interference term is responsible for the oscillatory intensity profile (see Fig. 1). The amplitude of the wakefield has a maximum at $w_{z}=2$, and in this case $C_{\beta, \max }=\frac{1}{2} \sqrt{e^{-1} \pi / 2} \approx 0.38$. Note, that for the case of a single mode, $\{m=0, n=0\}$, where the interference term vanishes, one recovers the usual linear theory solution for the plasma wakefield driven by a Gaussian laser pulse [43].

Magnetic fields, which are proportional to $a_{0}^{4}$ [49], are neglected in the linear case $a_{0}<1$, and the electric fields are given by $\mathbf{E}=-\nabla \phi(\zeta, x, y)$, where the gradient is taken in $(\zeta, x, y)$ coordinates, and the electric field is normalized to $m c^{2} k_{p} / e$. Assuming $k_{p}$ is constant, the transverse wakefield given by:

$$
\begin{aligned}
\boldsymbol{E}(\zeta, \boldsymbol{r}, \tau)= & 2 C_{\beta} \sin \zeta \sum_{\nu} a_{\nu}^{2} \eta_{\nu}^{2} \chi_{\nu} \boldsymbol{\kappa}_{\nu} \\
& +\sin \zeta \sum_{\nu \neq \lambda} C_{u, \nu, \lambda} a_{\nu} a_{\lambda} \eta_{\nu} \eta_{\lambda}\left(\chi_{\nu} \boldsymbol{\kappa}_{\lambda}+\chi_{\lambda} \boldsymbol{\kappa}_{\nu}\right) \\
& \cdot \cos \left(\Omega_{\nu, \lambda} \tau-\Delta \varphi_{\nu, \lambda}\right),
\end{aligned}
$$

where $\kappa_{i}=\left(\kappa_{x, i}, \kappa_{y, i}\right)$ with $i=(\nu, \lambda)$, and:

$$
\begin{aligned}
\kappa_{x, i}= & -\partial \chi_{i} / \partial x \\
= & \frac{H_{n_{i}}(y / \sqrt{R})}{\sqrt{R}}\left(H_{m_{i}+1}(x / \sqrt{R})\right. \\
& \left.-\frac{x}{\sqrt{R}} H_{m_{i}}(x / \sqrt{R})\right) e^{-\frac{x^{2}+y^{2}}{2 R}}, \\
\kappa_{y, i}= & -\partial \chi_{i} / \partial y \\
= & \frac{H_{m_{i}}(x / \sqrt{R})}{\sqrt{R}}\left(H_{n_{i}+1}(y / \sqrt{R})\right. \\
& \left.-\frac{y}{\sqrt{R}} H_{n_{i}}(y / \sqrt{R})\right) e^{-\frac{x^{2}+y^{2}}{2 R}},
\end{aligned}
$$

The exponential terms in Eqs. (8) and (9) decrease the amplitude of the field in transverse directions and can be neglected near the $\hat{z}$ axis or for a wide laser pulse. Then, the field of Eq. (7) becomes approximately harmonic if the two beating modes have different parity, e.g., even and odd modes. The polarization of the oscillation of the wakefield can be controlled by the laser pulse amplitude ratio and the phase. As examples, the simple cases of linear and circular polarization are discussed as following.

\section{A. Linear oscillation of wakefield}

Considering two modes, $\left\{m=0, n=0, a=a_{0}\right\}$ and $\left\{m=1, n=0, a=a_{1}\right\}$ with the same initial phase $\Delta \varphi=0$ and wavelength $\Delta M_{p, 0,1}=0$ which gives $C_{\beta}=C_{u, 0,1}$, the near-axis wakefield is given by: 


$$
\begin{aligned}
E_{x}(\zeta, x, \tau) \simeq & \frac{2 C_{\beta}}{R}\left(a_{0}^{2}-2 a_{1}^{2}\right) \sin \zeta \cdot x \\
& -C_{\beta} \sqrt{\frac{2}{R}} a_{0} a_{1} \sin \zeta \cdot \cos (\Omega \tau), \\
E_{y}(\zeta, y, \tau) \simeq & \frac{2 C_{\beta}}{R} a_{0}^{2} \sin \zeta \cdot y, \\
E_{z}(\zeta, y, \tau) \simeq & -C_{\beta}\left(a_{0}^{2}+\frac{2 x^{2}}{R} a_{1}^{2}\right) \cos \zeta \\
& -C_{\beta} \sqrt{\frac{2}{R}} a_{0} a_{1} \cos \zeta \cdot x \cdot \cos (\Omega \tau)
\end{aligned}
$$

where the exponential and higher-order of $x / \sqrt{R}$ are neglected by the assumption of a wide laser pulse, $x^{2}+y^{2} \ll R$, and $\Omega=\Omega_{0,1}=\Omega_{H}$. One can see that the wakefield in Eq. (10) linearly oscillates in the $\hat{x} \hat{z}$ plane which provides an additional driving force for an injected electron. As a result, the oscillation of the electron is linearly polarized in the same plane as shown later in Sec. IV.

\section{B. Circular oscillation of wakefield}

Considering three modes, $\left\{m=0, n=0, a=a_{0}, \varphi=0\right\}$, $\left\{m=1, n=0, a=a_{1}, \varphi=0\right\}, \quad$ and $\quad\{m=0, p=1$, $\left.a=a_{2}, \varphi=\pi / 2\right\}$, the near-axis wakefield is then given with the same assumptions above as:

$$
\begin{aligned}
E_{x}(\zeta, x, \tau) \simeq & \frac{2 C_{\beta}}{R}\left(a_{0}^{2}-2 a_{1}^{2}\right) \sin \zeta \cdot x \\
& -C_{\beta} \sqrt{\frac{2}{R}} a_{0} a_{1} \sin \zeta \cdot \cos (\Omega \tau), \\
E_{y}(\zeta, y, \tau) \simeq & \frac{2 C_{\beta}}{R}\left(a_{0}^{2}-2 a_{2}^{2}\right) \sin \zeta \cdot y \\
& -C_{\beta} \sqrt{\frac{2}{R}} a_{0} a_{2} \sin \zeta \cdot \sin (\Omega \tau), \\
E_{z}(\zeta, y, \tau) \simeq & -C_{\beta}\left[a_{0}^{2}+\frac{2}{R}\left(a_{1}^{2} x^{2}+a_{2}^{2} y^{2}\right)\right] \cos \zeta \\
& -C_{\beta}\left[\sqrt{\frac{2}{R}} a_{0} a_{1} \cdot x \cdot \cos \Omega \tau\right. \\
& +\left(\frac{2}{R} a_{1} a_{2} \cdot x y+\sqrt{\frac{2}{R}} a_{0} a_{2} \cdot y\right) \\
& \cdot \sin \Omega \tau] \cos \zeta .
\end{aligned}
$$

One can see that the oscillation of wakefield is circularly polarized in the transverse plane if $a_{1}=a_{2}$ near the axis of a plasma channel, and the ellipticity of oscillation can be controlled by the difference of the laser strength, $a_{1}$ and $a_{2}$.
As seen from Eq. (10) and (11), if the condition:

$$
a_{0}^{2}-2 a_{1}^{2}=0 \quad \text { or } \quad a_{0}^{2}-2 a_{2}^{2}=0
$$

is satisfied, the electric field is independent of the spatial coordinates near the propagation axis $\hat{z}$. Note that, in such a case, the oscillation is only circularly polarized in the transverse plane for the case of three beating modes. As shown in Fig. 2(a), the projection of the transverse wakefield on the $\hat{x} \hat{z}$ plane, $E_{x}$, is generated by two different modes without the condition (12). The electron in such a wakefield undergoes both betatron and undulator oscillations and can be treated in the same way as in our previous work $[37,40,41]$, where the electron beam principally undergoes an envelope oscillation due to the betatron focusing force. Such oscillation will give rise to the spread of momentum and energy of the electron beam, and subsequently broaden the radiation spectrum as discussed later. In order to alleviate this limit, matching demands are made on the injection conditions of the electron beam. Unfortunately, such injection conditions are currently difficult to realize. Through application of the matching
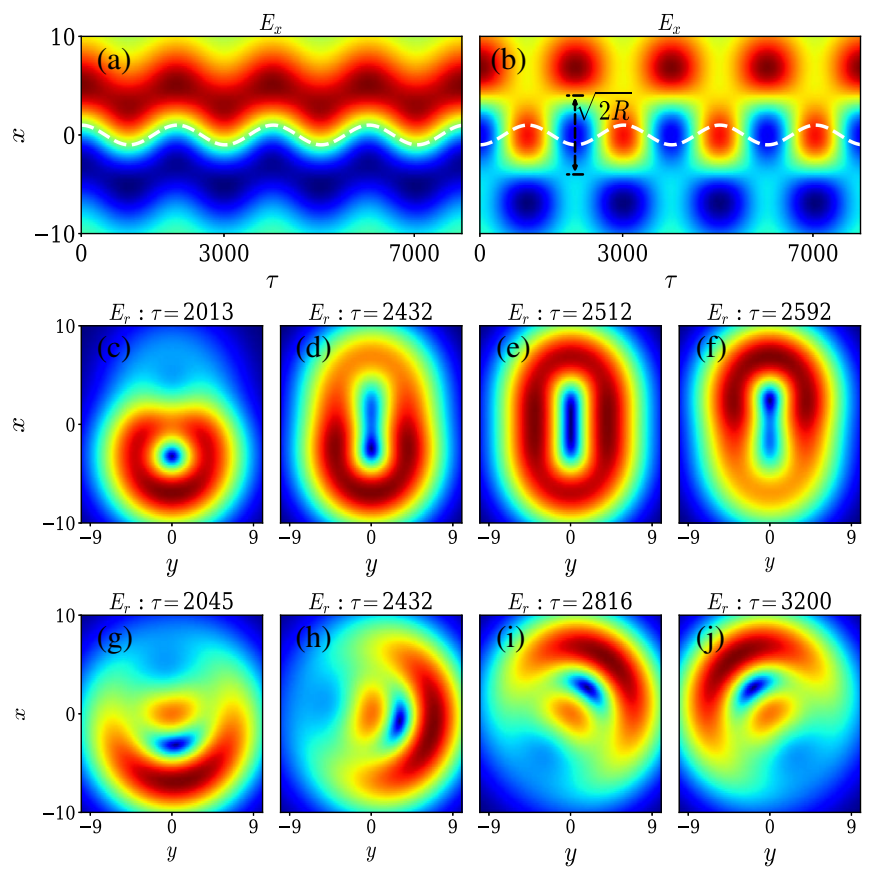

FIG. 2. (a), (b) Projection of the transverse wakefield $E_{x}$, generated by two different modes without and with the condition (12), as a function of time $\tau$ on $\hat{x} \hat{z}$ plane. The dashed white lines show the trajectories of an electron in the wakefield; (c), (d), (e), (f) Slices of the transverse wakefield $E_{r}$, generated by two different modes with the condition (12), on $\hat{x} \hat{y}$ plane at four different propagation positions, $\tau=2269$, 2432, 2512, 2592, respectively; (g), (h), (i), (j) Slices of transverse wakefield $E_{r}$, generated by three different modes with the condition (12), at four different propagation positions, $\tau=2045,2432$, 2816, 3200, respectively. 
conditions (12), the transverse wakefield becomes harmonic near the $\hat{z}$ axis, $|x|<\sqrt{R / 2}$ as shown in Fig. 2(b), which releases the requirements of the initial injection conditions since there is no focusing force anymore. As a result, the electron beam maintains good quality during propagation inside such a wakefield. This is very beneficial for the generation of a narrowband radiation spectrum for electron beam experiments.

We indicate that significant inaccuracy in the real experimental conditions, such as errors on the laser strength and plasma density channel, may cause the condition (12) to be unfulfilled. Therefore, as seen from Eqs. (10) and (11), the matching condition given by Eq. (12) required for restriction in this regime demands very small variation of the laser field strength parameters and plasma density. For example, a variation of the first mode is given by $a_{1}^{\prime}=a_{1}+\delta a_{1}$, where $a_{1}$ satisfies the condition in Eq. (12) with $a_{0}$. This variation will lead to the betatron oscillation of an injected electron of a gamma factor $\gamma_{0}$ with the frequency

$$
\Omega_{\delta \beta}=\Omega_{\beta, 1} \sqrt{\frac{\delta a_{1}}{a_{1}}\left(2+\frac{\delta a_{1}}{a_{1}}\right)},
$$

where the injection phase is at $\zeta=-3 \pi / 2$ and $\Omega_{\beta, 1}=$ $\sqrt{4 C_{\beta} a_{1}^{2} /\left(\gamma_{0} R\right)}$ is the single mode-introduced betatron oscillation frequency. The frequency is scaled as a function of $\delta a_{1} / a_{1}$ in Fig. 3. This oscillation gives an additional lowfrequency peak in the radiation spectrum same as discussed in our previous work [37,41]. With an uncertainty in plasma density channel $\delta n / \Delta n$ significantly high, $\Delta n^{\prime}=\Delta n+\delta n$, the high-order term may appear in the equation of the transverse field in Eqs. (10) and (11). For example, in the linearly polarized case, the additional high-order term is

$$
E_{x}(\zeta, x, \tau)_{\delta n} \simeq \frac{4 C_{\beta} a_{1}^{2}}{R^{2}}\left(1+\frac{\delta n}{\Delta n}\right)^{2} \sin \zeta \cdot x^{3}
$$

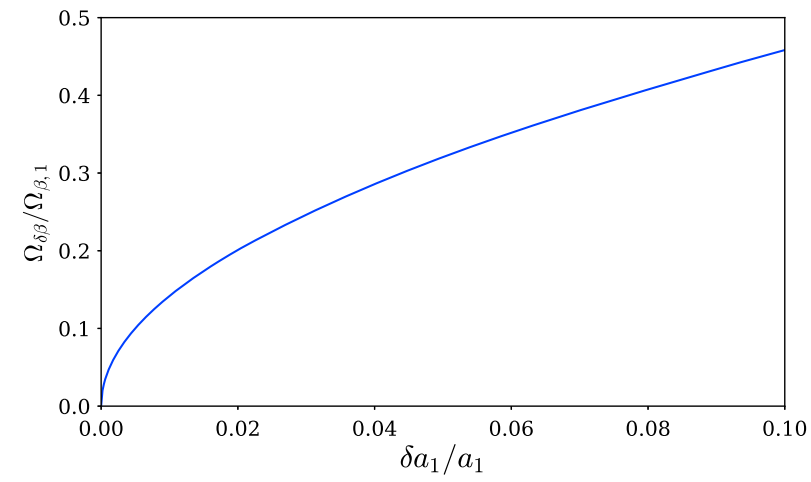

FIG. 3. Betatron oscillation frequency of an injected electron in Eq. (13), introduced by the inaccuracy of laser strength, $a_{1}$.
This turns the dynamics of an injected electron into a Duffing oscillator [50] and then destroys the oscillation. Here, an analytic solution is not available and the superposition principle is also no longer valid. To avoid this situation, the uncertainty in plasma density channel is required as $\delta n \ll \Delta n$, and, at the same time, the wide laser pulse is assumed by

$$
\frac{x^{2}}{R}\left(1+\frac{\delta n}{\Delta n}\right) \ll 1
$$

As a result, the term in Eq. (14) is negligible in the transverse wakefield near the channel axis.

The slices of the transverse wakefield $E_{r}=\sqrt{E_{x}^{2}+E_{y}^{2}}$ generated by two and three modes at four different propagation positions with the matching condition (12) are shown in Fig. 2(c)-(f) and (g)-(j) respectively. For the case of two modes, the wakefield linearly oscillates only in the $\hat{x}$ direction and is guided in $\hat{y}$ direction. For the case of three modes, the wakefield rotates around the propagation $\hat{z}$ axis. As a result, an electron injected into such a wakefield undergoes an oscillation with the same polarization of the oscillation of the wakefield.

\section{DYNAMICS OF THE ELECTRONS}

For simplicity, the wakefield with linear oscillation in the $\hat{x} \hat{z}$ plane is considered and the circular case can be treated in the same way. With the condition Eq. (12), the transverse electric field for the phase $\zeta=-3 \pi / 2$, is given by:

$$
E_{x}(x, \tau)=E_{0} \cos (\Omega \tau),
$$

where $E_{z}=0$ and $E_{0}=C_{u} a_{0} a_{1} \sqrt{2 / R}$ is the amplitude of the wakefield. For a relativistic electron injected on-axis with Lorentz factor $\gamma_{0} \gg 1$, the motion is described by a linear harmonically driven oscillator equation:

$$
\frac{d u_{x}}{d \tau}=-E_{0} \cos (\Omega \tau) .
$$

By assuming $u_{x}(\tau=0)=0$ where the electron is injected without angle, the solution is given by:

$$
u_{x}=-a_{u} \sin (\Omega \tau) \text {, }
$$

where $u_{x}=p_{x} / m_{e} c$ is the normalized transverse momentum in the $\hat{x}$ direction and $a_{u}=E_{0} / \Omega$ is the oscillation strength. Notice that the electron does not oscillate in the $\hat{y} \hat{z}$-plane if it is initially injected on-axis $y(\tau=0)=0$ and without an angle in the $\hat{y}$-direction, i.e., $u_{y}(\tau=0)=0$. Equation (18) is the exact solution for electrons propagating on the $\hat{z}$ axis and is capable of describing a tightly focused electron beam propagating near the axis of the plasma channel because there is no betatron oscillation in 


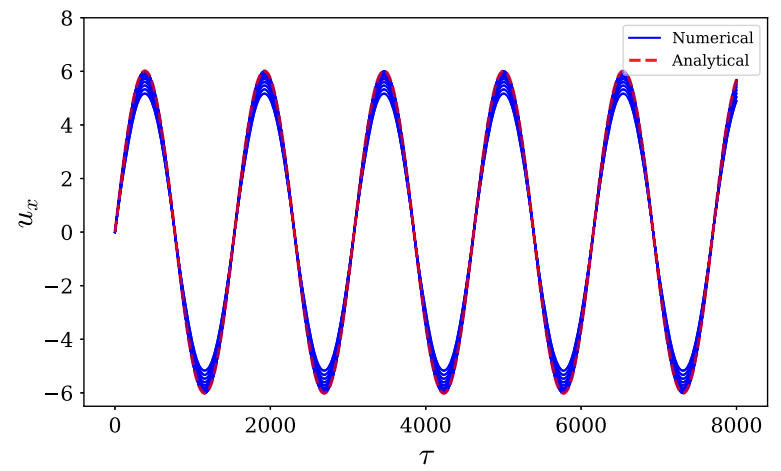

FIG. 4. The normalized momentum $u_{x}$ in $\hat{x}$ direction. Red dashed line: analytical result from Eq. (18); Blue solid lines: numerical results obtained for an electron beam by solving the transverse momentum equation with the electric field in Eq. (7).

such a wakefield. In Fig. 4, the red dashed line is the analytical result of a single on-axis electron from Eq. (18), and the blue solid lines are numerical results obtained for a cylindrically symmetric electron beam with a spot size of $\sigma_{b}=4.0$ by solving the transverse momentum equation with the electric field in Eq. (7). The normalized emittance of the electron beam is 0.0047 . The energy spread is neglected and the beam is assumed to be infinitely small in the longitudinal direction purely for an illustrative purpose. The wakefield is generated by beating two linearly polarized laser pulses, $\left\{m=0, n=0, a=0.56, r_{m}=6, \varphi=0\right\}$ and $\left\{m=1, n=0, a=0.4, r_{m}=6, \varphi=0\right\}$. The on-axis numerical result of $u_{x}$ agrees exactly with the analytical result in Eq. (18). The attenuation of off-axis results is caused by the exponential term in Eq. (7). For a wide laser pulse, this decrement is slight.

With a small inaccuracy $\delta a_{1}$ in the laser pulse strength as discussed in Sec. III, the undulator strength is perturbed by

$$
\frac{\delta a_{u}}{a_{u}}=\frac{1}{\Omega^{2} / \Omega_{\delta \beta}^{2}-1},
$$

where $\Omega_{\beta, 1}$ is given in Eq. (13). As shown in Fig. 5, the variation of the undulator strength increases with a larger $\delta a_{1} / a_{1}$. It is also seen that high laser pulse strength $a_{1}$ gives faster growth rate of the variation $\delta a_{u} / a_{u}$ for the same $\delta a_{1} / a_{1}$. This indicates that the more accurate control is required for the experiments with higher laser pulse strength. The uncertainty of plasma density channel can also introduce the variation of undulator strength, scaled as $\delta a_{u} / a_{u} \propto(1+\delta n / \Delta n)^{2}$.

\section{UNDULATOR RADIATION}

Radiation emitted by a single relativistic electron oscillating in a plasma-based undulator field with constant oscillation strength, $a_{u}$, has been extensively studied [41]. In our current scheme, the oscillation strength $a_{u}$ can be tuned over a broad range, $0<a_{u}<\gamma_{0} \Omega \sqrt{2 R}$. As a result, it

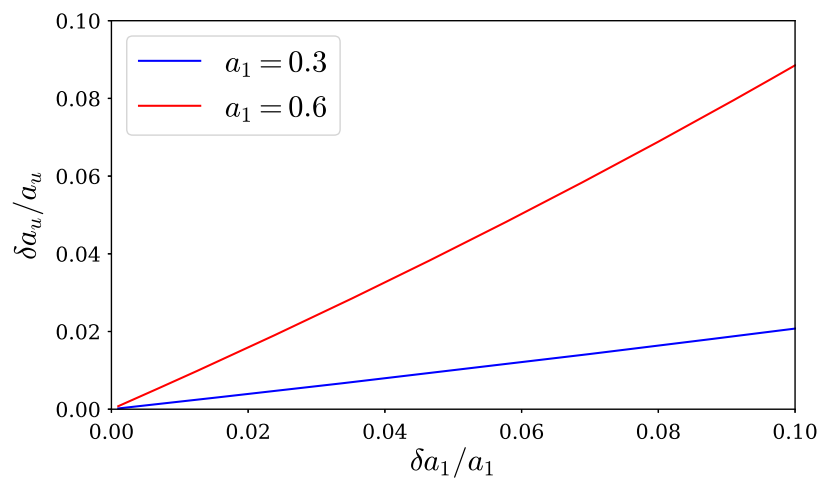

FIG. 5. Variation of the undulator strength with different laser pulse strength: read solid line with $a_{1}=0.6$; blue solid line with $a_{1}=0.3$. The parameters are chosen as: plasma density $10^{18} \mathrm{~cm}^{-3}$, wavelength of the laser pulse $\lambda_{L}=1 \mu \mathrm{m}$ and laser spot size $r_{m}=1.14$, gamma factor of the injected electron $\gamma_{0}=1000$.

is capable of generating a narrow $\mathrm{x}$-ray radiation spectrum for $a_{u}<1$ as well as synchrotronlike $\mathrm{x} / \gamma$-ray radiation for $a_{u}>1$.

\section{A. Radiation spectrum with narrow bandwidth}

The radiation spectrum of an single electron can be calculated from Lienard-Wiechert potentials [51] by using the transverse Eq. (18) and longitudinal trajectories $u_{z} \approx u_{z 0}-\delta(\tau) \sin (\zeta+\Delta \zeta)$ where $\delta(\tau)=$ $C_{u} a_{0} a_{1} x \sqrt{2 / R} \sin (\Omega \tau) / \Omega$. The phase slippage could be approximately given as $\Delta \zeta \approx\left(\beta_{e}-\beta_{p}\right) \tau$ with $\beta_{p} \approx$ $1-1 / M_{p}$ being the phase velocity of plasma wave and $\beta_{e} \approx 1$ being the longitudinal speed of electron. It is seen that there is no acceleration on axis in the condition of Eq. (12), which will relieve the stress from dephasing and, thus, benefit the generation of a narrow spectrum. The depletion of the laser pulse occurs after approximately a dephasing length. Therefore, in the case of a low plasma density and tightly focused electron beam, effects of dephasing of the electron and laser pulse depletion could be neglected for a short propagation distance relative to the dephasing length $L_{d} \approx 2 \pi M_{p}^{2}$ [43] and $u_{z} \approx u_{z 0}$ for the injection phase $\zeta=-3 \pi / 2$. The near-axis photon energyangular spectrum of the fundamental emission for a linear polarized oscillation and small undulator strength $a_{u}<1$ has been calculated in [41] with such a trajectory. The central frequency is $\omega_{c e}=2 \gamma_{0}^{2} \Omega /\left(1+a_{u}^{2} / 2\right)$ and the natural bandwidth is given by $\Delta \omega / \omega=1 / N_{u}$, where $N_{u}$ is the number of oscillation periods.

Consider a plasma channel of on-axis density $n_{0}=$ $10^{18} \mathrm{~cm}^{-3}$ and a Gaussian electron beam with parameters such as $\zeta=-3 \pi / 2$, total charge $Q=1 \mathrm{pC}$, transverse radius in $\hat{x}$ direction $x_{b}=0.1$, temporal length $\Delta \tau_{b}=0.2$, energy $\gamma_{0}=1000$ and energy spread $\sigma_{\gamma} / \gamma_{0}=1 \%$. The normalized transverse emittance of the elctron beam 

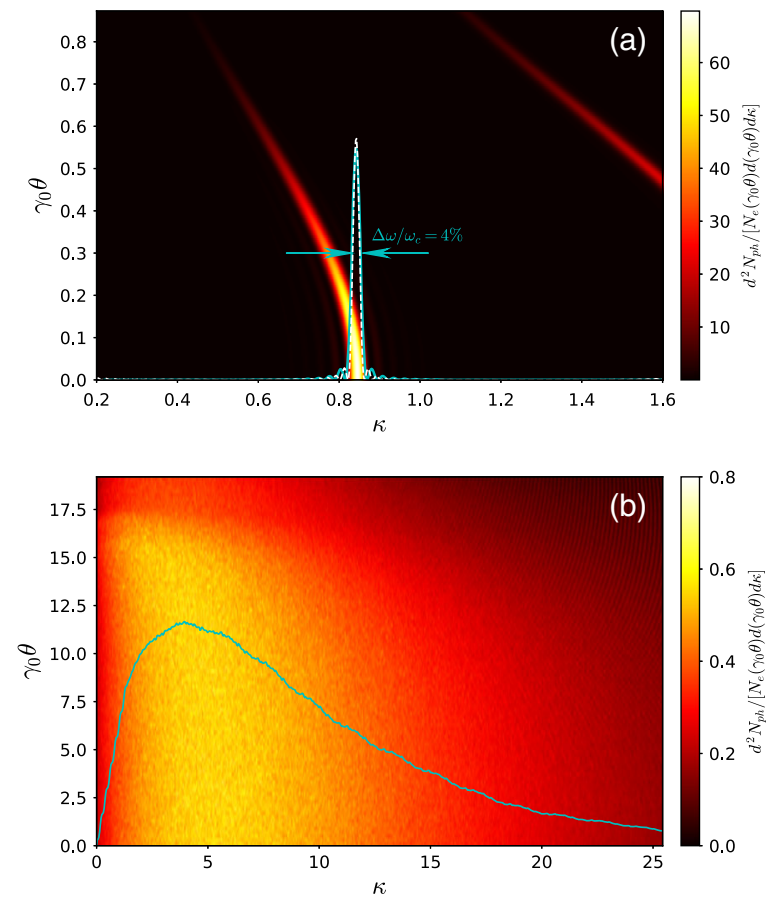

FIG. 6. Radiation spectrum from plasma-based undulator/wiggler with (a) $a_{u} \simeq 0.62$ and (b) $a_{u} \simeq 16$. Dashed white line is the theoretical on-axis solution and solid cyan lines in both plots are for the numerical on-axis radiation with $\gamma_{0} \theta=0$.

is 0.0047. Such an electron beam can be obtained from a laser wakefield accelerator [5]. The radiation is calculated by Virtual Detector for Synchrotron Radiation [52] simulation. Figure 6(a) shows the narrow x-ray radiation spectrum $d^{2} N_{p h} /\left[N_{e}\left(\gamma_{0} \theta\right) d\left(\gamma_{0} \theta\right) d \kappa\right]$ generated by two beating laser pulses $\{m=0, n=0, a=$ $\left.0.21, r_{m}=1.14, \varphi=0\right\} \quad$ and $\quad\{m=1, p=0, a=0.15$, $\left.r_{m}=1.14, \varphi=0\right\}$ after $n_{u}=30$ periods of oscillations, where the total oscillation length is much smaller than the dephasing length $L_{d} \approx 7000 . N_{p h}$ is the number of emitted photons and $N_{e}$ is the number of electrons inside the beam. The azimuthal angle is normalized as $\gamma_{0} \theta$ and the frequency is $\kappa=\omega /\left(2 \gamma_{0}^{2} \Omega\right)$. The oscillation strength is $a_{u} \simeq 0.62$. The solid cyan line shows the numerical on-axis radiation spectrum of the electron beam at $\gamma_{0} \theta=0$. The central frequency is $\kappa=0.83\left(\hbar \omega_{c e}=2.93 \mathrm{keV}\right)$ with a full-widthhalf-maximum (FWHM) bandwidth of $\Delta \omega / \omega=4 \%$. It mainly benefits from the matching condition in Eq. (12) which eliminates the betatron focusing force near the axis of the plasma channel as seen in our previous work [41].

The spectrum of the electron beam is broadened with respect to the theoretical natural bandwidth of an single electron due to the spread of energy and undulator strength which could be estimated as [53],

$$
\left[\frac{\Delta \omega}{\omega}\right]_{\mathrm{FWHM}} \approx \sqrt{\left(\frac{1}{N_{u}}\right)^{2}+\left(\frac{2 \Delta \gamma}{\gamma_{0}}\right)^{2}+\left(\frac{\Delta a_{u}}{4 \gamma_{0}}\right)^{2}},
$$

where $\Delta \gamma$ is from the initial energy spread as well as the energy chirp caused by the finite duration of the electron beam and contributes an extra $0.5 \%$ broadening in our case. $\Delta a_{u}$ mainly comes from the phase slippage of electrons and the finite duration of the electron beam, and contributes an additional $0.2 \%$ broadening. The experimental inaccuracy of laser strength and plasma density channel can also introduce the additional broadening of the spectrum due to the variation of $a_{u}$ as given in Eq. (19), but is neglected here.

\section{B. Synchrotronlike broad radiation spectrum}

For large $a_{u}>1$, this scheme is also capable of generating a synchrotronlike spectrum with the critical frequency of $\omega_{c r}=(3 / 2) \gamma_{0}^{2} E_{0}=(3 / 2) \gamma_{0}^{2} c_{u} a_{0} a_{1} / r_{m}$. Figure 6(b) shows the synchrotron-like hard $\mathrm{x}$-ray radiation spectrum generated by two beating laser pulses $\left\{m=0, n=0, a=0.84, r_{m}=\right.$ $1.14, \varphi=0\}$ and $\left\{m=1, n=0, a=0.6, r_{m}=1.14, \varphi=0\right\}$. The oscillation strength is $a_{u} \simeq 16$. The critical frequency is $\kappa=5.45\left(\hbar \omega_{p}=19 \mathrm{keV}\right)$.

\section{Harmonics with OAM}

The circular motion of an electron in the circularly polarized wakefield Eq. (11) with the condition Eq. (12) is given as

$$
\begin{aligned}
& u_{x}=-a_{u} \sin (\Omega \tau), \\
& u_{y}=a_{u} \cos (\Omega \tau), \\
& u_{z}=u_{z 0},
\end{aligned}
$$

where on-axis injection is assumed, $u_{x, y}(\tau=0)=0$. The radiated field is given by the Lienard-Wiechert potentials and then the radiation intensity in an interaction duration $T$ can be directly derived from Jackson's formula [51] as

$$
\frac{d^{2} I}{d \Omega_{s a} d \omega}=M\left|\sum_{n=-\infty}^{+\infty} J_{n}(\alpha \sin \theta) e^{-i n \pi / 2} \frac{u_{z 0} T}{2} \vec{I}_{n}\right|^{2}
$$

where $\Omega_{s a}$ is the solid angle, $M=e^{2} \omega^{2} /\left(4 \pi c \gamma_{0}^{2}\right)$ and $\alpha=a_{u} \omega /\left(\gamma_{0} \Omega\right)$ are defined variables, and $\vec{I}_{n}$ describes the flux field of the emitted photon beam. The exponential term $e^{-i n \pi / 2}$ is due to the initial conditions of motion. Please see more details of the derivation in supplemental material [54]. In the case where all the electrons inside a beam are in the same initial phase as described by Eq. (21), the radiation intensity reaches its maximum. With the trajectory in Eq. (21) and the unit vector to the observer $\hat{n}=\sin \theta \cos \phi \cdot \hat{e}_{x}+\sin \theta \sin \phi \cdot \hat{e}_{y}+\cos \theta \cdot \hat{e}_{z}$, the flux field for $n$th harmonic is given as

$$
\vec{I}_{n}=I_{n+} \hat{e}_{+}+I_{n-} \hat{e}_{-}+I_{n z} \hat{e}_{z}
$$



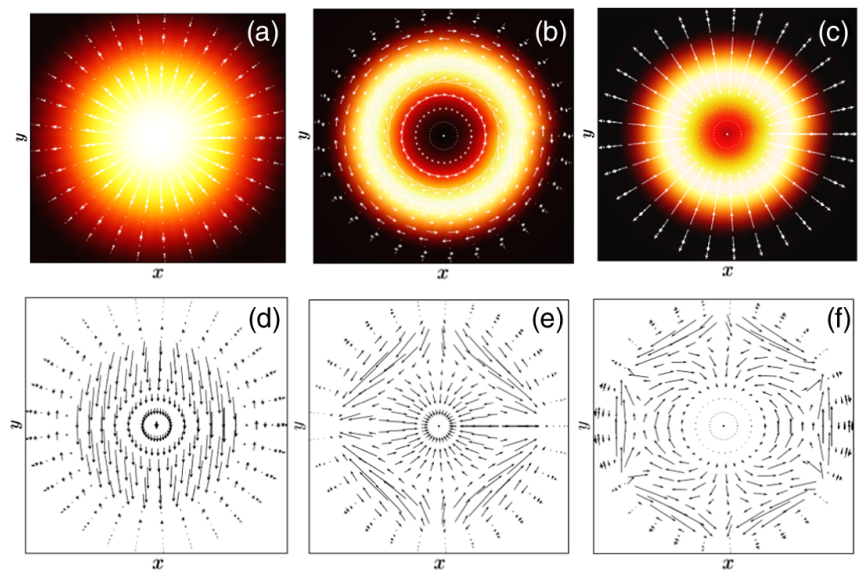

FIG. 7. (a)-(c) show the intensity and transverse vector field (white arrows, $\vec{I}_{\perp}=I_{n+} \hat{e}_{+}+I_{n-} \hat{e}_{-}$) of radiation for the fundamental $(n=0)$, second $(n=1)$, and third $(n=2)$ harmonics, respectively in Eq. (24) and (25), viewed along z-axis; (d)-(f) show the corresponding rotation components.

where $\hat{e}_{ \pm}=\left(\hat{e}_{x} \pm i \hat{e}_{y}\right) / \sqrt{2}$ are the vertex direction view from $z$-axis and the transverse components are given as

$$
\begin{aligned}
I_{n+}= & \frac{1}{\sqrt{2}}\left[i K S_{n+1} e^{i n \phi}\left(2-\sin ^{2} \theta\right)\right. \\
& \left.+i K S_{n-1} e^{i(n-2) \phi} \sin ^{2} \theta+S_{n} e^{-i \phi} \sin 2 \theta\right],
\end{aligned}
$$

and

$$
\begin{aligned}
I_{n-}= & \frac{1}{\sqrt{2}}\left[-i K S_{n-1} e^{i n \phi}\left(2-\sin ^{2} \theta\right)\right. \\
& \left.-i K S_{n+1} e^{i(n+2) \phi} \sin ^{2} \theta+S_{n} e^{-i \phi} \sin 2 \theta\right],
\end{aligned}
$$

where $S_{n}=\sin c((\nu-n \Omega) T / 2), \nu=\omega\left(1-\beta_{z 0} \cos \theta\right)$, and $K=a_{u} / u_{z 0}$ is the pitch angle of electron motion. The first two terms in the right-hand side (RHS) of Eq. (24) and (25) contribute to field vortex and the last terms to the polarization. As shown in Fig. 7, the fundamental harmonic, in (a) and (d), does not show any vortex and therefore does not carry any OAM. However, the vortex nature is clearly shown in the higher-order harmonics, for example, in (b), (c), (e), and (f), which then could carry the OAM. It is noticed that the field does not rotate in the even harmonics due to the compensation of the last terms in the RHS of Eq. (24) and (25), as shown in Figs. 7(a) and 7(c).

\section{CONCLUSIONS AND DISCUSSION}

In this paper, a scheme of plasma undulator/wiggler produced by the beating of several Hermite-Gaussian laser pulses with different modes in a parabolic plasma channel is further discussed. The oscillation of the excited plasma wakefield is caused by the interference between each set of two pulses. The oscillation frequency is then determined by the difference of the parameters of modes, $\Omega_{\nu, \lambda}=\theta_{\nu}-\theta_{\lambda}$, including mode number $(m, n)$ and wavelength $M_{p,(m, n)}$. From the nonparaxial wave equation of the laser pulse and linear laser-plasma wakefield theory, the undulator field is derived for the beating of several Hermite-Gaussian laser pulses in the Cartesian geometry. The polarization of the field is also discussed for a special case. An initial matching condition of pulse strength is given to produce a harmonic field without a focusing force near the propagation axis. As a result, there is no betatron oscillation for an electron beam injected into such a field and therefore the quality of the emitted photon beam is improved. The dynamics as well as the radiation of an injected electron are studied in the linear case. The oscillation strength $a_{u}=E_{0} / \Omega \propto a_{0} a_{1} r_{m}$ is in a wide range and is capable of producing a plasma-based undulator field for a narrow $\mathrm{x}$-ray source or a wiggler field for a broad synchrotronlike $\mathrm{x} / \gamma$-ray source with higherorder emitted harmonics containing OAM. In comparison to $[40,41]$ where the oscillation strength is determined by the off-set of initial injection position of laser pulse, the current scheme is much easier to experimentally implement. The most important feature of the current scheme is its ability to overcome previous limitations on the generation of higher-energy radiation in the linear plasma wakefield [40].

\section{ACKNOWLEDGMENTS}

This work was supported by the Helmholtz Association (Young Investigator's Group VH-NG-1037), by the NSFC (11674341), and by the Director, Office of Science, Office of High Energy Physics, of the U.S. Department of Energy under Contract No. DE-AC02-05CH11231. The authors are grateful to D. Seipt, I. Andriyash, S. Kuschel, and M. Zolotorev for fruitful discussions. Authors would like to acknowledge the usage of the open-access visualization software MATPLOTLIB [55]. The Authors acknowledge the usage of the Skoltech CDISE HPC cluster Zhores for obtaining the results presented in this paper [56].

[1] S. Mangles, C. Murphy, Z. Najmudin, A. Thomas, J. Collier, A. Dangor, E. Divall, P. Foster, J. Gallacher, C. Hooker, D. Jaroszynski, A. Langley, W. Mori, P. Norreys, F. Tsung, R. Viskup, B. Walton, and K. Krushelnick, Monoenergetic beams of relativistic electrons from intense laser-plasma interactions, Nature (London) 431, 535 (2004).

[2] C. Geddes, C. Toth, J. V. Tilborg, E. Esarey, C. Schroeder, D. Bruhwiler, C. Nieter, J. Cary, and W. Leemans, Highquality electron beams from a laser wakefield accelerator using plasma-channel guiding, Nature (London) 431, 538 (2004).

[3] W. P. Leemans, B. Nagler, A. J. Gonsalves, C. Tóth, K. Nakamura, C. G. R. Geddes, E. Esarey, C. B. Schroeder, 
and S. M. Hooker, GeV electron beams from a centimetrescale accelerator, Nat. Phys. 2, 696 (2006).

[4] E. Kallos, T. Katsouleas, W. D. Kimura, K. Kusche, P. Muggli, I. Pavlishin, I. Pogorelsky, D. Stolyarov, and V. Yakimenko, High-Gradient Plasma-Wakefield Acceleration with Two Subpicosecond Electron Bunches, Phys. Rev. Lett. 100, 074802 (2008).

[5] W. T. Wang, W. T. Li, J. S. Liu, Z. J. Zhang, R. Qi, C. H. Yu, J. Q. Liu, M. Fang, Z. Y. Qin, C. Wang, Y. Xu, F. X. Wu, Y. X. Leng, R. X. Li, and Z. Z. Xu, High-Brightness High-Energy Electron Beams from a Laser Wakefield Accelerator via Energy Chirp Control, Phys. Rev. Lett. 117, 124801 (2016).

[6] J. van Tilborg, S. Steinke, C. G. R. Geddes, N. H. Matlis, B. H. Shaw, A. J. Gonsalves, J. V. Huijts, K. Nakamura, J. Daniels, C. B. Schroeder, C. Benedetti, E. Esarey, S. S. Bulanov, N. A. Bobrova, P. V. Sasorov, and W. P. Leemans, Active Plasma Lensing for Relativistic Laser-PlasmaAccelerated Electron Beams, Phys. Rev. Lett. 115, 184802 (2015).

[7] S. Kuschel, D. Hollatz, T. Heinemann, O. Karger, M. B. Schwab, D. Ullmann, A. Knetsch, A. Seidel, C. Rödel, M. Yeung, M. Leier, A. Blinne, H. Ding, T. Kurz, D. J. Corvan, A. Sävert, S. Karsch, M. C. Kaluza, B. Hidding, and M. Zepf, Demonstration of passive plasma lensing of a laser wakefield accelerated electron bunch, Phys. Rev. Accel. Beams 19, 071301 (2016).

[8] C. Thaury, E. Guillaume, A. Dpp, R. Lehe, A. Lifschitz, K. T. Phuoc, J. Gautier, J.-P. Goddet, A. Tafzi, A. Flacco, F. Tissandier, S. Sebban, A. Rousse, and V. Malka, Demonstration of relativistic electron beam focusing by a laserplasma lens, Nat. Commun. 6, 6860 (2015).

[9] R. Tatchyn, Variable-period electrostatic and magnetostatic undulator designs for generating polarized soft $\mathrm{x}$ rays at PEP, Rev. Sci. Instrum. 60, 2571 (1989).

[10] V. Papadichev, An electrostatic undulator with singlepolarity feed, Nucl. Instrum. Methods Phys. Res., Sect. A 429, 377 (1999).

[11] S. Bellucci, S. Bini, V. M. Biryukov, Y. A. Chesnokov, S. Dabagov, G. Giannini, V. Guidi, Y. M. Ivanov, V. I. Kotov, V. A. Maisheev, C. Malagù, G. Martinelli, A. A. Petrunin, V. V. Skorobogatov, M. Stefancich, and D. Vincenzi, Experimental Study for the Feasibility of a Crystalline Undulator, Phys. Rev. Lett. 90, 034801 (2003).

[12] U. I. Uggerh $\varnothing j$, The interaction of relativistic particles with strong crystalline fields, Rev. Mod. Phys. 77, 1131 (2005).

[13] T. N. Wistisen, K. K. Andersen, S. Yilmaz, R. Mikkelsen, J. L. Hansen, U. I. Uggerhøj, W. Lauth, and H. Backe, Experimental Realization of a New Type of Crystalline Undulator, Phys. Rev. Lett. 112, 254801 (2014).

[14] S. Tantawi, M. Shumail, J. Neilson, G. Bowden, C. Chang, E. Hemsing, and M. Dunning, Experimental Demonstration of a Tunable Microwave Undulator, Phys. Rev. Lett. 112, 164802 (2014).

[15] C. Joshi, T. Katsouleas, J. Dawson, Y. Yan, and J. Slater, Plasma wave wigglers for free-electron lasers, IEEE J. Quantum Electron. 23, 1571 (1987).

[16] I. Andriyash, R. Lehe, A. Lifschitz, C. Thaury, J.-M. Rax, K. Krushelnick, and V. Malka, An ultracompact X-ray source based on a laser-plasma undulator, Nat. Commun. 5, 4736 (2014).

[17] M. Zolotorev, Laser driven attosecond SASE X-ray FEL, Nucl. Instrum. Methods Phys. Res., Sect. A 483, 445 (2002).

[18] P. Sprangle, B. Hafizi, and J. Peñano, Laser-pumped coherent X-ray free-electron laser, Phys. Rev. Accel. Beams 12, 050702 (2009).

[19] A. Bacci, M. Ferrario, C. Maroli, V. Petrillo, and L. Serafini, Transverse effects in the production of $\mathrm{x}$ rays with a free-electron laser based on an optical undulator, Phys. Rev. Accel. Beams 9, 060704 (2006).

[20] P. Balcou, Proposal for a Raman X-ray free electron laser, Eur. Phys. J. D 59, 525 (2010).

[21] I. a. Andriyash, P. Balcou, and V. T. Tikhonchuk, Collective properties of a relativistic electron beam injected into a high intensity optical lattice, Eur. Phys. J. D 65, 533 (2011).

[22] I. a. Andriyash, E. D'Humières, V. T. Tikhonchuk, and P. Balcou, Betatron emission from relativistic electrons in a high intensity optical lattice, Phys. Rev. Accel. Beams 16, 100703 (2013).

[23] A. D. Debus, M. Bussmann, M. Siebold, A. Jochmann, U. Schramm, T. E. Cowan, and R. Sauerbrey, Traveling-wave Thomson scattering and optical undulators for high-yield EUV and X-ray sources, Appl. Phys. B 100, 61 (2010).

[24] J. E. Lawler, J. Bisognano, R. A. Bosch, T. C. Chiang, M. A. Green, K. Jacobs, T. Miller, R. Wehlitz, D. Yavuz, and R. C. York, Nearly copropagating sheared laser pulse FEL undulator for soft X-rays, J. Phys. D 46, 325501 (2013).

[25] C. Chang, C. Tang, and J. Wu, High-Gain ThompsonScattering X-Ray Free-Electron Laser by Time-Synchronic Laterally Tilted Optical Wave, Phys. Rev. Lett. 110, 064802 (2013).

[26] E. Hemsing, A. Marinelli, and J. B. Rosenzweig, Generating Optical Orbital Angular Momentum in a High-Gain Free-Electron Laser at the First Harmonic, Phys. Rev. Lett. 106, 164803 (2011).

[27] E. Hemsing, A. Knyazik, M. Dunning, D. Xiang, A. Marinelli, C. Hast, and J. B. Rosenzweig, Coherent optical vortices from relativistic electron beams, Nat. Phys. 9, 549 (2013).

[28] M. Katoh, M. Fujimoto, H. Kawaguchi, K. Tsuchiya, K. Ohmi, T. Kaneyasu, Y. Taira, M. Hosaka, A. Mochihashi, and Y. Takashima, Angular Momentum of Twisted Radiation from an Electron in Spiral Motion, Phys. Rev. Lett. 118, 094801 (2017).

[29] J. Vieira, J. T. Mendonça, and F. Quéré, Optical Control of the Topology of Laser-Plasma Accelerators, Phys. Rev. Lett. 121, 054801 (2018).

[30] M. F. Andersen, C. Ryu, P. Cladé, V. Natarajan, A. Vaziri, K. Helmerson, and W. D. Phillips, Quantized Rotation of Atoms from Photons with Orbital Angular Momentum, Phys. Rev. Lett. 97, 170406 (2006).

[31] M. Babiker, C. R. Bennett, D. L. Andrews, and L. C. Dávila Romero, Orbital Angular Momentum Exchange in the Interaction of Twisted Light with Molecules, Phys. Rev. Lett. 89, 143601 (2002).

[32] R. Juchtmans and J. Verbeeck, Orbital angular momentum in electron diffraction and its use to determine chiral crystal symmetries, Phys. Rev. B 92, 134108 (2015). 
[33] J. T. Mendonça, B. Thidé, and H. Then, Stimulated Raman and Brillouin Backscattering of Collimated Beams Carrying Orbital Angular Momentum, Phys. Rev. Lett. 102, 185005 (2009).

[34] A. M. Yao and M. J. Padgett, Orbital angular momentum: origins, behavior and applications, Adv. Opt. Photonics 3, 161 (2011).

[35] N. B. Simpson, K. Dholakia, L. Allen, and M. J. Padgett, Mechanical equivalence of spin and orbital angular momentum of light: an optical spanner, Opt. Lett. 22, 52 (1997).

[36] P. Roth, G. K. L. Wong, R. Beravat, C. M. Harvey, M. H. Frosz, R. Sopalla, and P. St.J. Russell, Measurement of the orbital angular momentum spectrum in twisted coreless photonic crystal fiber, in Conference on Lasers and Electro-Optics (Optical Society of America, San Jose, California, 2018), p. SW3K.2, http://dx.doi.org/10.1364/ CLEO_SI.2018.SW3K.2.

[37] S. G. Rykovanov, C. B. Schroeder, E. Esarey, C. G. R. Geddes, and W. P. Leemans, Plasma Undulator Based on Laser Excitation of Wakefields in a Plasma Channel, Phys. Rev. Lett. 114, 145003 (2015).

[38] M. Chen, J. Luo, F.-Y. Li, F. Liu, Z.-M. Sheng, and J. Zhang, Tunable synchrotron-like radiation from centimeter scale plasma channels, Light: Science and Applications 5, e16015 (2016).

[39] J. W. Wang, C. B. Schroeder, R. Li, M. Zepf, and S. G. Rykovanov, Plasma channel undulator excited by highorder laser modes, Sci. Rep. 7, 16884 (2017).

[40] B. Lei, J. Wang, V. Kharin, M. Zepf, and S. Rykovanov, $\gamma$-Ray Generation from Plasma Wakefield Resonant Wiggler, Phys. Rev. Lett. 120, 134801 (2018).

[41] S. G. Rykovanov, J. W. Wang, V. Y. Kharin, B. Lei, C. B. Schroeder, C. G. R. Geddes, E. Esarey, and W. P. Leemans, Phys. Rev. Accel. Beams 19, Tunable polarization plasma channel undulator for narrow bandwidth photon emission, 090703 (2016).

[42] C. G. R. Geddes, C. Toth, J. van Tilborg, E. Esarey, C. B. Schroeder, J. Cary, and W. P. Leemans, Guiding of Relativistic Laser Pulses by Preformed Plasma Channels, Phys. Rev. Lett. 95, 145002 (2005).

[43] E. Esarey, C. B. Schroeder, and W. P. Leemans, Physics of laser-driven plasma-based electron accelerators, Rev. Mod. Phys. 81, 1229 (2009).

[44] A. J. Gonsalves, T. P. Rowlands-Rees, B. H. P. Broks, J. J. A. M. van der Mullen, and S. M. Hooker, Transverse
Interferometry of a Hydrogen-Filled Capillary Discharge Waveguide, Phys. Rev. Lett. 98, 025002 (2007).

[45] T. P. A. Ibbotson et al., Laser-wakefield acceleration of electron beams in a low density plasma channel, Phys. Rev. Accel. Beams 13, 031301 (2010).

[46] L. Landau and E. Lifshitz, Quantum Mechanics, NonRelativistic Theory: Volume 3 (Course of Theoretical Physics Series), 3rd ed. (Butterworth-Heinemann, Washington, D.C., 1977).

[47] E. Cormier-Michel, E. Esarey, C. G. R. Geddes, C. B. Schroeder, K. Paul, P. J. Mullowney, J. R. Cary, and W. P. Leemans, Control of focusing fields in laser-plasma accelerators using higher-order modes, Phys. Rev. Accel. Beams 14, 031303 (2011).

[48] W. Lu, C. Huang, M. M. Zhou, W. B. Mori, and T. Katsouleas, Limits of linear plasma wakefield theory for electron or positron beams, Phys. Plasmas 12, 063101 (2005).

[49] L. Gorbunov, P. Mora, and T. M. Antonsen, Jr., Magnetic Field of a Plasma Wake Driven by a Laser Pulse, Phys. Rev. Lett. 76, 2495 (1996).

[50] H. J. Korsch, H.-J. Jodl, and T. Hartmann, Chaos (Springer-Verlag, Berlin Heidelberg, 2008).

[51] J. Jackson, Classical Electrodynamics (John Wiley \& Sons, New York, 1975).

[52] M. Chen, E. Esarey, C. Geddes, C. Schroeder, G. Plateau, S. Bulanov, S. Rykovanov, and W. Leemans, Modeling classical and quantum radiation from laser-plasma accelerators, Phys. Rev. Accel. Beams 16, 030701 (2013).

[53] S. G. Rykovanov, C. G. R. Geddes, J.-L. Vay, C. B. Schroeder, E. Esarey, and W. P. Leemans, Quasimonoenergetic femtosecond photon sources from Thomson Scattering using laser plasma accelerators and plasma channels, J. Phys. B 47, 234013 (2014).

[54] See Supplemental Material at http://link.aps.org/ supplemental/10.1103/PhysRevAccelBeams.22.071302 the detailed derivation of the radiated flux field in Eq. (22) from a circular motion of a single electron generated by the proposed scheme.

[55] J. D. Hunter, Matplotlib: A 2D Graphics Environment, Comput. Sci. Eng. 9, 90 (2007).

[56] I. Zacharov, R. Arslanov, M. Gunin, D. Stefonishin, S. Pavlov, O. Panarin, A. Maliutin, S. G. Rykovanov, and M. Fedorov, arXiv:1902.07490. 